\title{
Review Article \\ Serum Lipid Profiles and Cancer Risk in the Context of Obesity: Four Meta-Analyses
}

\author{
Jennifer C. Melvin, ${ }^{1,2}$ Lars Holmberg, ${ }^{1,2,3}$ Sabine Rohrmann, ${ }^{4}$ \\ Massimo Loda, ${ }^{5,6,7,8}$ and Mieke Van Hemelrijck ${ }^{1}$ \\ ${ }^{1}$ Cancer Epidemiology Group, Division of Cancer Studies, King's College London, School of Medicine, 3rd Floor, Bermondsey Wing, \\ Guy's Hospital, London SE1 9RT, UK \\ ${ }^{2}$ Regional Cancer Centre, Uppsala University Hospital, 75185 Uppsala, Sweden \\ ${ }^{3}$ Department of Surgical Sciences, Uppsala University, 75185 Uppsala, Sweden \\ ${ }^{4}$ Department of Epidemiology and Prevention of Cancer, Institute of Social and Preventative Medicine, \\ University of Zurich, 8001 Zurich, Switzerland \\ ${ }^{5}$ Department of Medical Oncology, Dana-Farber Cancer Institute, Boston, MA 02215, USA \\ ${ }^{6}$ Department of Pathology, Brigham and Women's Hospital of Harvard Medical School, Boston, MA 02115, USA \\ ${ }^{7}$ Broad Institute of Harvard and MIT, 7 Cambridge Center, Cambridge, MA 02142, USA \\ ${ }^{8}$ Division of Cancer Studies, School of Medicine, King's College London, 3rd Floor, Bermondsey Wing, \\ Guy's Hospital, London SE1 9RT, UK
}

Correspondence should be addressed to Jennifer C. Melvin; jennifer.melvin@kcl.ac.uk

Received 16 August 2012; Revised 26 October 2012; Accepted 2 November 2012

Academic Editor: Thomas E. Rohan

Copyright (C) 2013 Jennifer C. Melvin et al. This is an open access article distributed under the Creative Commons Attribution License, which permits unrestricted use, distribution, and reproduction in any medium, provided the original work is properly cited.

The objective here was to summarize the evidence for, and quantify the link between, serum markers of lipid metabolism and risk of obesity-related cancers. PubMed and Embase were searched using predefined inclusion criteria to conduct meta-analyses on the association between serum levels of TG, TC, HDL, ApoA-I, and risk of 11 obesity-related cancers. Pooled relative risks (RRs) and 95\% confidence intervals were estimated using random-effects analyses. 28 studies were included. Associations between abnormal lipid components and risk of obesity-related cancers when using clinical cutpoints (TC $\geq 6.50 ; \mathrm{TG} \geq 1.71 ; \mathrm{HDL} \leq 1.03$; ApoA-I $\geq 1.05 \mathrm{mmol} / \mathrm{L}$ ) were apparent in all models. RRs were 1.18 (95\% CI: 1.08-1.29) for TC, 1.20 (1.07-1.35) for TG, 1.15 (1.01-1.32) for HDL, and 1.42 (1.17-1.74) for ApoA-I. High levels of TC and TG, as well as low levels of HDL and ApoA-I, were consistently associated with increased risk of obesity-related cancers. The modest RRs suggest serum lipids to be associated with the risk of cancer, but indicate it is likely that other markers of the metabolism and/or lifestyle factors may also be involved. Future intervention studies involving lifestyle modification would provide insight into the potential biological role of lipid metabolism in tumorigenesis.

\section{Introduction}

Obesity is a major worldwide problem, over $30 \%$ of adults in Western populations are obese, and there is growing evidence of the associated health risks associated [1-5]. The link between obesity and cancer risk has been studied extensively, but the results of individual studies do not suggest a consistent association $[1,6,7]$. Common cancers studied in the context of obesity include colorectal, breast, prostate, endometrial, pancreatic, liver, ovarian, kidney, gallbladder, leukaemia, and oesophageal cancers [4, 7-17].

The underlying mechanisms of action are not clear $[1,18-$ 20]. A solid understanding could translate directly to patient benefit through implementation of therapeutic strategies to reduce cancer risk and mortality [19]. Assuming that the lipid metabolism plays a role in the biological processes driving the development of cancer, this could be easily modified by existing methods such as exercise, medication, or diet. 
Increased physical activity levels improve cardiovascular and overall mortality in healthy populations [21-24]. Also, physical activity after cancer diagnosis is associated with improvements in cancer outcomes [25, 26] and metabolic markers such as cholesterol $[27,28]$. As such, improvements in lipid levels through uptake of physical activity may also translate into improvements in cancer-specific survival. Experimental evidence largely suggests that statins, a commonly used drug to lower cholesterol levels, reduce cancer risk, though further trials are needed [29]. However, a large meta-analysis by the Cholesterol Treatment Trialists' Collaboration showed no statistically significant associations between statin use and cancer risk [30]. Nevertheless, due to heterogeneity of plasma lipid profiles in overweight and obese people, there may be some inconsistency in the associations for serum lipids and cancer risk [31]. Moreover, study populations were often small, insufficient information was collected (e.g., lack of BMI measurement, few lipid components measured), and timing of blood sampling in relation to diagnosis varied widely $[32,33]$. In addition, it is thought that tissue types are influenced differently by lipid components and to varying degrees $[2,32]$.

With these meta-analyses, we aimed to summarize and quantify the evidence for the link between markers of lipid metabolism and risk of obesity-related cancers. We examined the associations between four components of the lipid profile measured in serum (total cholesterol (TC), triglycerides (TGs), high-density lipoprotein (HDL), and apolipoprotein A-I (ApoA)) and risk of cancers previously shown to be linked with obesity.

\section{Methods}

2.1. Literature Search Strategy. We used computerised literature search databases (PubMed search followed by an Embase search) to identify full text and abstracts published to date. Searches were conducted both with and without MeSH terms ("neoplasms/epidemiology," "cancer," "hyperlipidemias," "lipoproteins, HDL," "hypertriglyceridemia," "lipoproteins, apo A"). Except for English language, human subjects, adults, and publications within the last 10 years no additional restrictions were added to the search. We also included "grey literature," such as letters and abstracts presented in relevant conference meetings. All references of the selected articles were checked, including hand searches.

2.2. Inclusion Criteria. The final collection of selected studies was chosen based on the following criteria: the publication pertained to an epidemiological study (cohort or case-control studies), which measured the serum concentration of at least one of the selected lipid components (TC, TGs, HDL, and ApoA-I) prior to cancer diagnosis; the analytical methods were well described, with sufficient data available; the cancers included must have previously been linked to an increased risk associated with obesity. Those included were colorectal, breast, prostate, endometrial, pancreatic, liver, ovarian, kidney, gallbladder, leukaemia, and oesophageal cancer [7]. To include studies with large enough power, only those with at least 20 cancer cases were included. Initially, titles and abstracts of articles were reviewed in order to ascertain whether they potentially fit the inclusion criteria. If there was doubt over whether an article met the relevant criteria, it was subjected to a thorough assessment. After this first selection, all articles underwent detailed evaluations of the methods and results. Figure 1 illustrates the study exclusion process.

2.3. Data Extraction. The following details were recorded for each study: author, year of publication, country where the study was undertaken, serum lipid component levels (mmol/l), study type (case-control or cohort), cancer type, number of cases and total subjects for each level of the lipid component(s) measured. To allow for ease of comparison, all values in conventional units $(\mathrm{mg} / \mathrm{dL})$ were converted into SI units (mmol/l) using conversion factors [34].

2.4. Statistical Methods. The effect of each lipid component on cancer risk was evaluated by calculating the random effects summary relative risk to allow for possible heterogeneity between study results. The analyses were conducted for dichotomized values of TC, TGs, HDL, and apoA-I). The following clinical cutpoints were used $\mathrm{TC} \geq 6.50$; $\mathrm{TG} \geq 1.71$; $\mathrm{HDL} \leq 1.03$; ApoA $-\mathrm{I} \leq 1.05 \mathrm{mmol} / \mathrm{L}$, all of which mirrored the NCEP and WHO guidelines as closely as practicable [35-39]. Some studies presented with dichotomised serum lipid levels, but for those that did not, participants from each study were divided into two groups based on their serum lipid level ("high" and "low"), and this mirrored the NCEP and WHO guidelines as closely as practicable. A first meta-analysis used all cancers from all studies. Potential heterogeneity of the study results was assessed with weighted forest plots, which display the relative risk estimates of cancer risk for each lipid component. Heterogeneity of the study results was also statistically evaluated using the $Q$-statistic as well as the $I^{2}$ statistic [40]. Sufficient data allowed for an individual analysis (using the methods described above) of prostate, colorectal, and breast cancers. Finally, we performed a metaregression to evaluate the effect of study design (i.e prospective cohort versus case-control studies). No other potential confounders were included in the metaregression due to the nature in which the data was presented through the papers. For example, some papers acknowledge having data for fasting status of the individuals at time of sampling, but did not provide the actual data by individuals. Potential publication bias and effect modification (by country and year) were assessed using Begg's Test and Egger's funnel plot. All analyses were performed using STATA (version 11.2).

\section{Results}

The initial searches produced a total of 701 articles. 33 studies were selected for further evaluation based on information from abstracts. Of these, 17 were excluded and a further 12 were added from hand searches and references of included studies, giving a total of 28 studies used in the primary data analysis. Eight studies were conducted in Asia, 12 in Europe, and eight in the USA (Table 1). All studies reported on at least one of the four lipid components under investigation. The nine included cancers were studied in association with cancer 


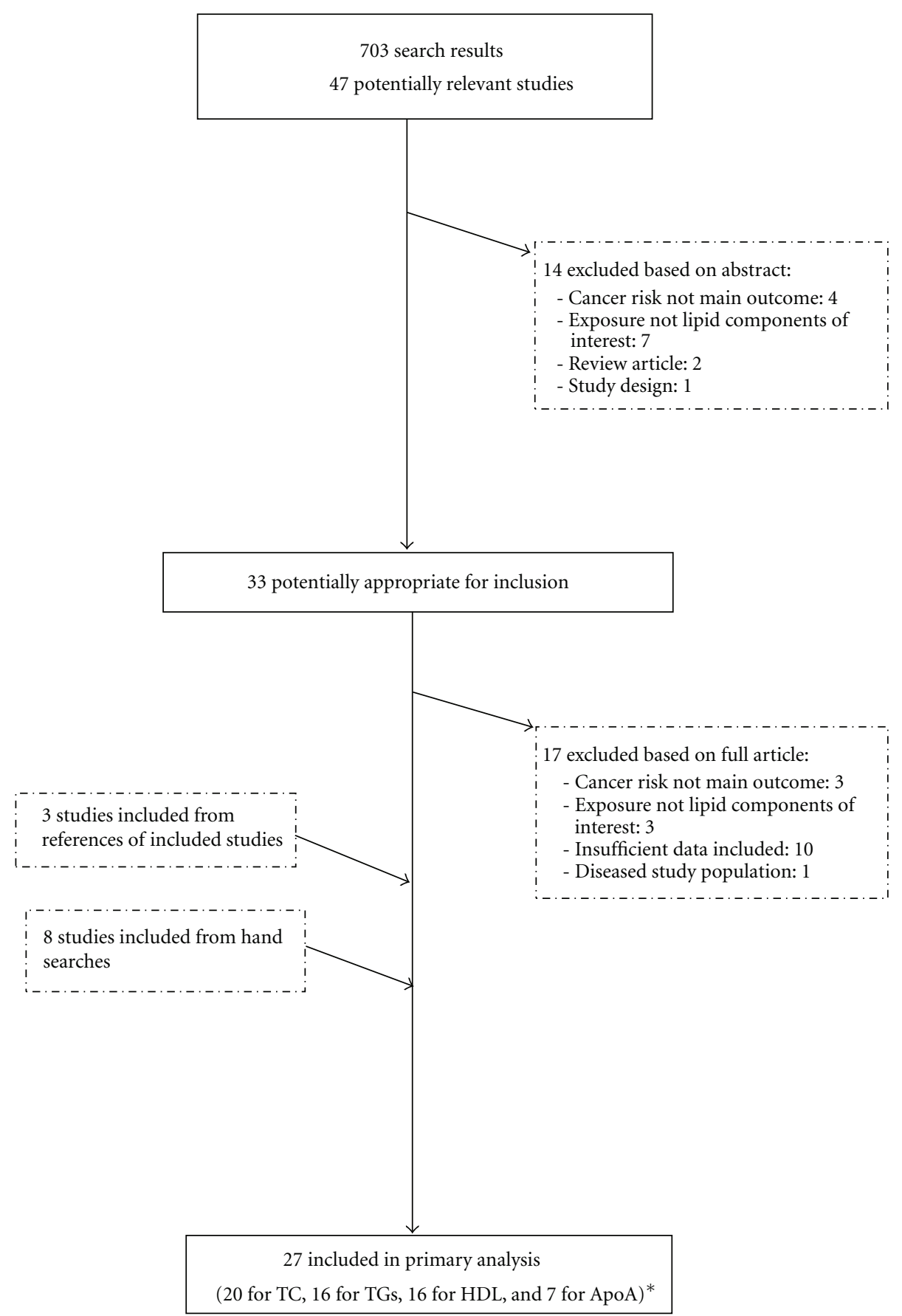

* NB: some studies were used in more than one analysis.

FIGURE 1

risk; all cancer diagnoses were histologically confirmed. Of those articles examined for inclusion, the major reasons for exclusion were missing information on methods and statistical analysis $(n=10)$, while a further three were excluded because serum lipid components were not the exposure of interest, and three were removed because incident cancer risk was not the outcome variable in the analysis.

For each lipid component studied we looked at the risk of developing cancer in those with abnormal versus normal levels. The random-effects analysis, comparing overall cancer risk and total cholesterol level, showed a pooled effects relative risk of 1.18 (95\% CI 1.08-1.29) (Figure 2). The Qstatistic and $I^{2}$-statistic suggested heterogeneity $(Q=250.02$; $\left.\mathrm{d} f=18 ; P=<0.000 ; I^{2}=92.8 \%\right)$, which warranted the use of a random-effects model. The association between TGs and overall cancer risk resulted in a pooled relative risk of 1.20 (95\% CI 1.07-1.35). The pooled relative risk was 1.15 (95\% CI 1.01-1.32) when studying the association between HDL 


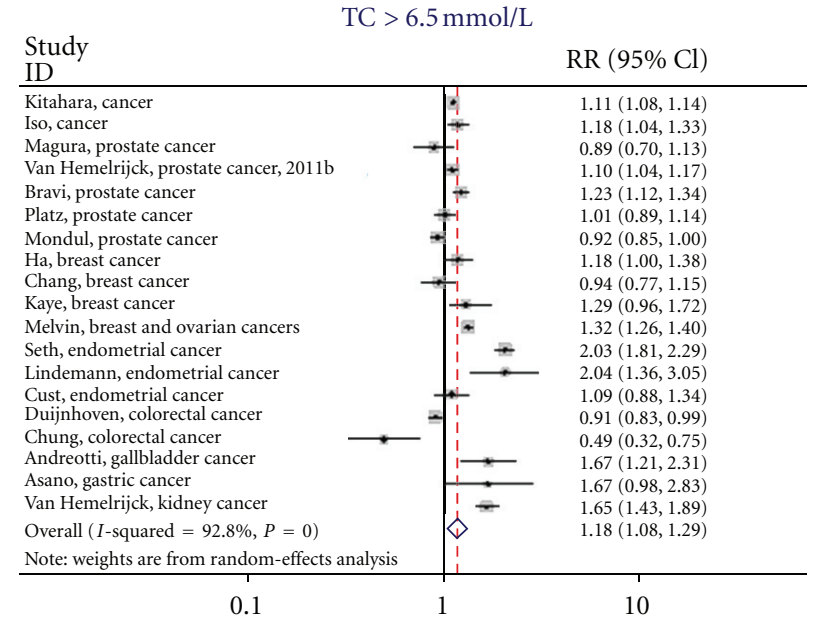

(a)

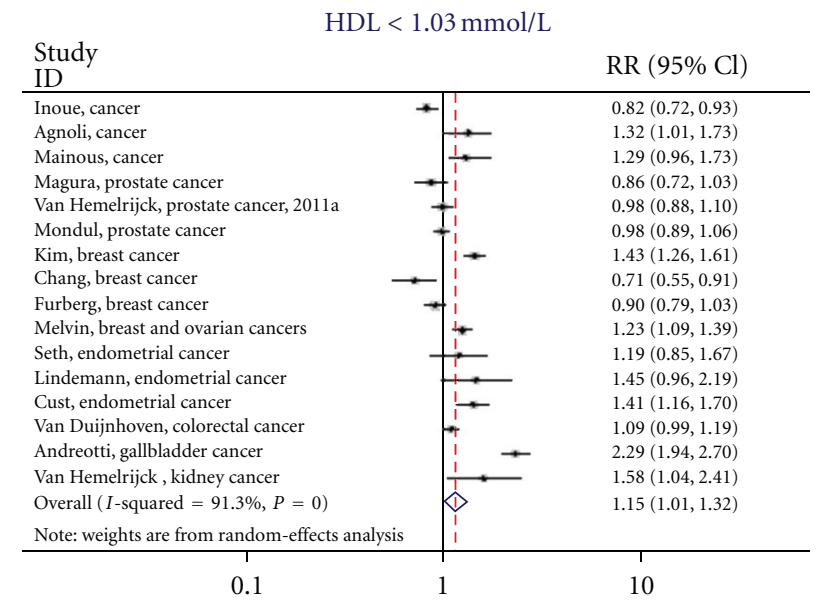

(c)

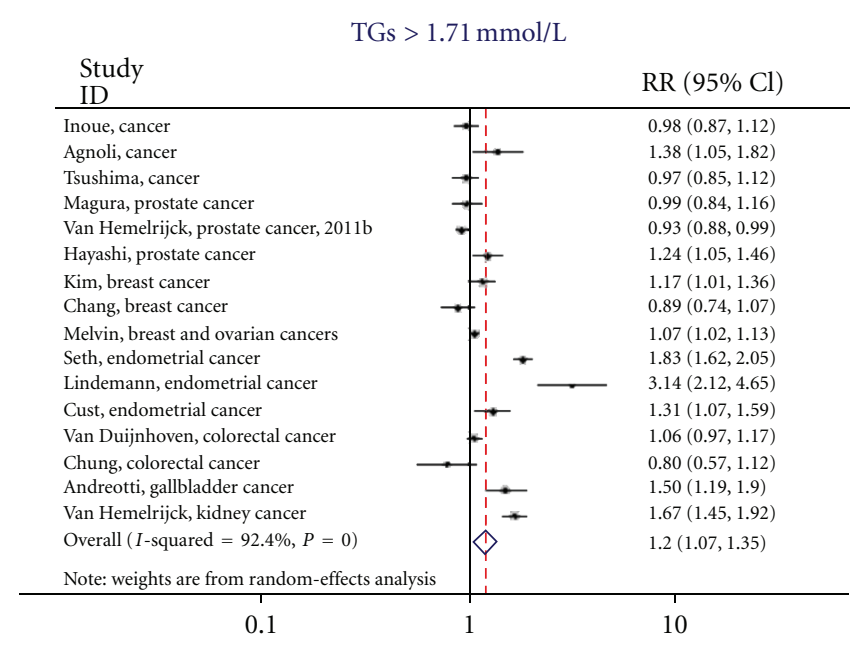

(b)

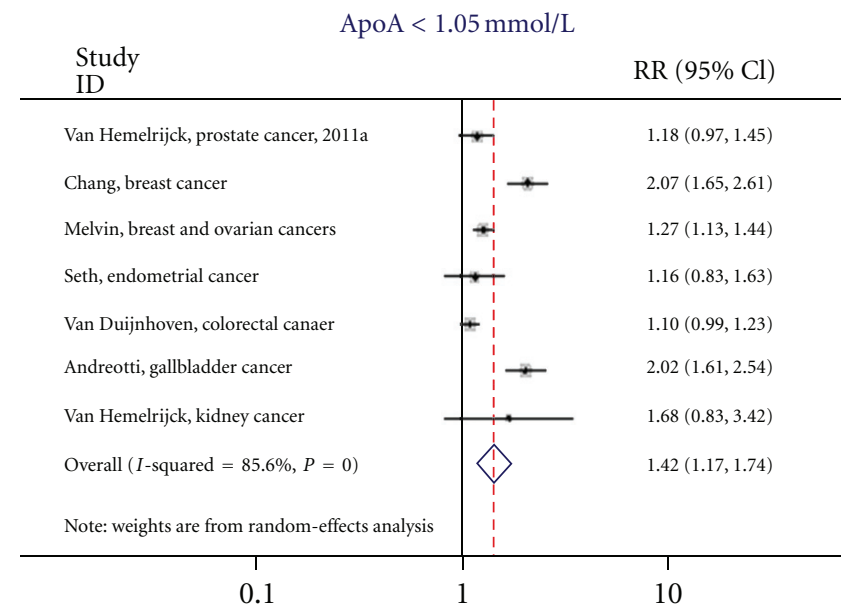

(d)

FIGURE 2: Individual forest for lipid components, the I-squared statistic is also illustrated in each plot—-total cholesterol; triglycerides; highdesity Lipoprotein; apolipoprotein A-I.

and risk of obesity-related cancers. The pooled effects relative risk of overall cancer was 1.42 (95\% CI 1.17-1.74) for ApoA-I (Figure 2).

We also conducted a stratified analysis by study type and found that the pooled RRs for case-control studies were slightly different (i.e., RR $(95 \% \mathrm{CI})=0.98(0.81-1.18))$ and $(\mathrm{RR}(95 \% \mathrm{CI})=1.20(1.04-1.38)$ for case-control and cohort studies on TC, resp.). We investigated this further with a metaregression, but did not find a statistically significant effect (i.e., $P$ value of 0.193 when studying TC). Begg's test did not indicate significant publication bias $(P=0.506)$, which is evident from the funnel plot, as there is a relatively symmetric distribution observed among studies with small sample size (Figure 3).

Finally, we also performed a meta-analysis specifically for TC and risk of prostate, breast, and colorectal cancer. The pooled relative risk for prostate cancer was 1.04 (95\% CI: 0.87-1.24), whereas it was 1.08 (95\% CI: 0.89-1.31) and 1.20 (95\% CI: 0.44-3.26) for breast and colorectal cancers, respectively.

\section{Discussion}

These meta-analyses summarize the current evidence for a link between serum markers of lipid metabolism and risk of obesity-related cancers. All pooled models showed evidence for an association between abnormal lipid components and risk of obesity-related cancers when using clinical cutpoints.

The precise aetiology of the link between obesity and risk of cancer has yet to be determined, but there has been growing evidence for a role of lipid metabolism in tumour development [18]. Apart from the studies listed on the link between serum lipids and cancer risk (Table 1), there is also preclinical evidence. For instance, it is thought that androgens stimulate prostate tumor growth via activation of pathways that regulate lipogenic gene expression, resulting in lipid accumulation [41]. Hyperlipidemia has also been shown to be involved in colorectal tumour development and initiation and progression of breast and prostate cancers [42-44]. Moreover, there is experimental evidence that fatty acid synthase (FAS), the enzyme that synthesizes fatty acids de novo, 


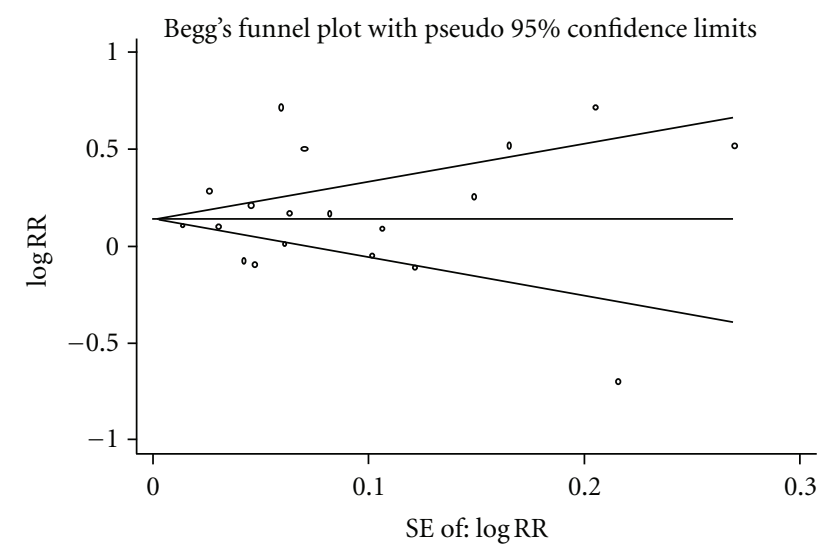

FIGURE 3: Funnel plot of Begg's test (with 95\% CI) to quantify presence of publication bias.

is involved in tumorigenesis [45-47]. For example, prostate cancers overexpressing FAS display aggressive behavior, with the highest expression in patients with bony metastatic disease $[47,48]$. In addition, nutritional studies showed that diets high in fat are linked to accelerated tumour growth and metastasis [42, 49]. Furthermore, cholesterol-lowering drugs such as statins have been shown to reduce the formation and spread of metastatic cancer cells $[50,51]$. Finally, the immune system is thought to play a role in the link between HDL, ApoA-I, and tumorigenesis [52]. These lipid components decrease free proinflammatory cytokines such as tumour necrosis factor- $\alpha$ (TNF- $\alpha$ ) which consequently reduces tissue damage, infiltration of macrophages and neutrophils, and attenuates tumour formation [53]. Therefore, low levels of HDL and Apo A-I may contribute to an inflammatory process linked to tumour biology.

Recent years have seen a multitude of reviews and metaanalyses comparing obesity and specific cancer risks; results varied widely depending on the type of cancer investigated, with relative risks ranging from 1.02 to 4.10 (breast cancer [11, 54-56], endometrial cancer [57], pancreatic [58-60], liver [17], prostate cancer [61], and colon and rectal cancer $[15,16])$. As a result, our findings for an association between serum lipid components and risk of cancer also varied by type of cancer, as can be seen from our results for HDL (Figure 2), show that there may be stronger correlation between serum HDL levels and cancer risk, dependent on cancer type. Those results focusing on a single cancer showed more consistent results, suggesting that even among obesity-related cancers there may be a different association with serum lipid levels.

4.1. Strengths and Limitations of This Study. The greatest strength of this study is that we examined four different components of the lipid profile in relation to risk of developing cancer in the context of obesity. We also made all possible efforts to include all relevant available publications, including searching the two main online databases (PubMed and Embase). Additionally, our clearly defined objective criteria for exposure, outcome, and other study characteristics were specified a priori. There was no evidence of publication bias in these analyses.
A number of the studies subdivided levels of lipid components, but this was not performed consistently across the studies. Studies which had not dichotomised serum lipid levels from the outset were divided into two groups based on their serum lipid level ("high" and "low") to mirror the NCEP and WHO guidelines as closely as practicable $[38,62]$. This crude categorization may have compromised the accuracy and resulted in miscategorising of individuals, but given the rather small differences in cutoffs we do not believe that this has had a major impact on our analyses.

Heterogeneity among studies may also arise from different method of assessment of serum lipids. By performing random-effects analyses, we have taken into account between-study variation. Within-person variation is a likely interference with results as the one measurement taken may not be representative for a person's average, or previous lipid levels. However, this variation will be present in all studies using a single measurement. In addition, adjustments made for confounding factors (e.g., gender or age) were not consistent across included studies and some sample sizes were relatively small or excluded one gender. Again, random effects analyses take into account this heterogeneity and in addition we included a metaregression analysis for study type.

In addition, the studies did not provide age-specific data, so it was not possible to conduct age-specific meta-analyses which presents us with a limitation. Persons younger than middle aged more rarely have abnormal lipid profiles and are also considerably less likely to be diagnosed with the cancers of interest than in those people aged over 50. Thus, this leaves our study population with a relatively low probability of having both sufficient exposure and number of cases in the lower age range. We do not believe that this will have had a major effect on our results, although it is worth considering that this may have diluted the strength of our findings somewhat.

Due to the information provided in the included studies, we had no means to adjust our analyses for cancer screening practices. Undoubtedly, these practices vary around the world and thus the differences could lead to the introduction of detection bias.

Finally, the analyses of the three individual cancers (prostate, breasts and colorectal cancers) did not produce statistically significant relative risks, which most likely follows from a lack of power due to the limited number of studies available for inclusion. Future research, with larger sample sizes, repeated measurements, and consistent adjustments for confounding could provide information to inform a more reliable estimate of links between serum lipid components and cancer risk.

4.2. Conclusions. Abnormal levels of all lipid components studied were statistically significantly associated with an increased risk of obesity-related cancers, with the strongest association for serum ApoA-I. Despite a suggestion for a link between the lipid metabolism and risk of cancer, the magnitude of the pooled relative risk was relatively small. This may be because the studied lipid components are markers of obesity or because they are markers of other lifestyle factors potentially associated with tumorigenesis. 


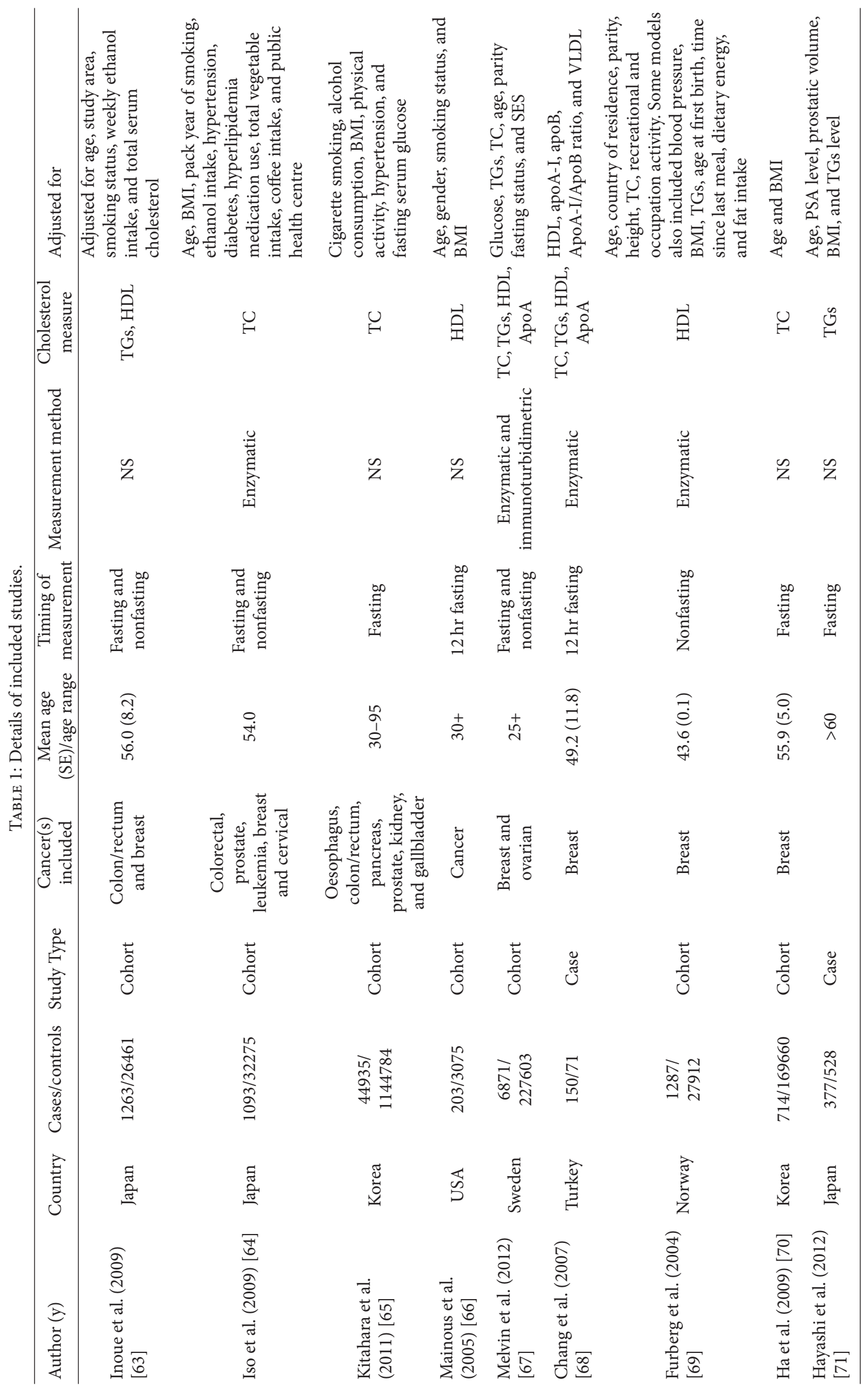




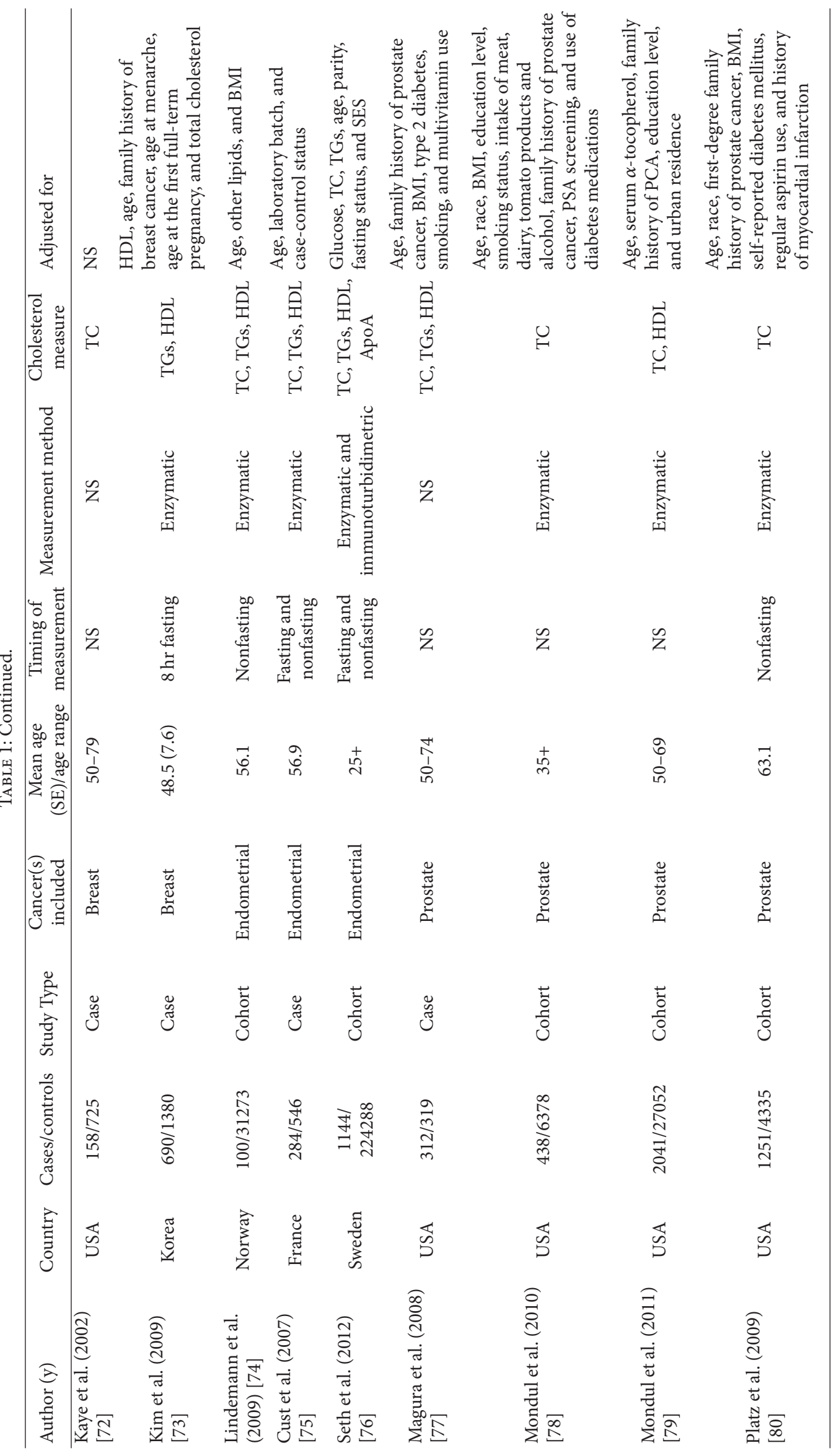




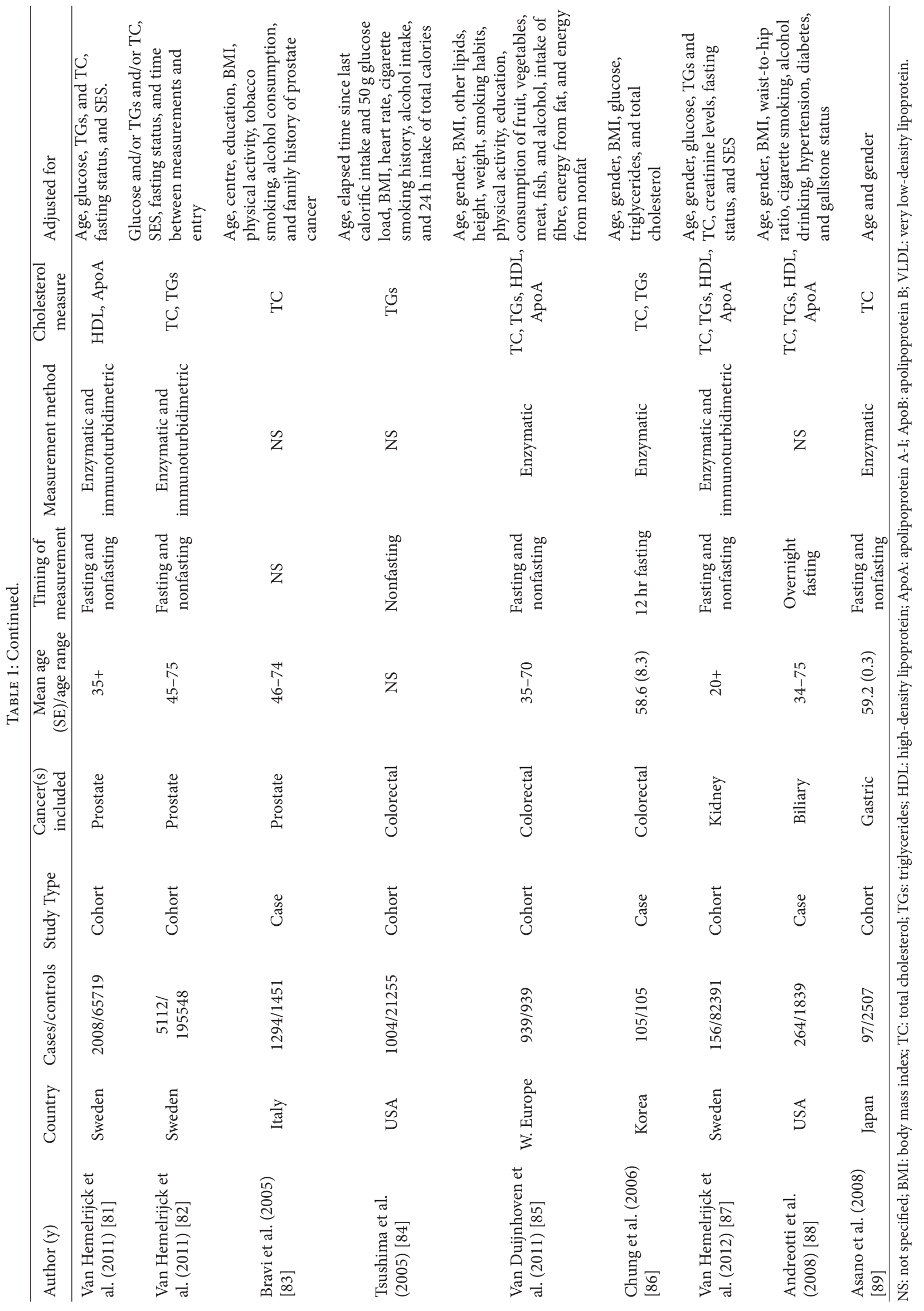


Since lipid components are easily modified through lifestyle interventions such as diet or exercise, research into serum lipid components and cancer risk presents a prime opportunity for intervention studies to help provide the desired insight into their biological role.

\section{Abbreviations \\ (TC): Total cholesterol \\ (TGs): Triglycerides \\ (HDL): High-density lipoprotein \\ (ApoA-I): Apolipoprotein A-I \\ (BMI): Body mass index.}

\section{Conflict of Interests}

The authors declare that they have no potential conflict of interests to disclose.

\section{Acknowledgments}

Funding was received from Cancer Research UK and the Swedish Cancer Society. This research was also supported by the Experimental Cancer Medicine Centre at King's College London and also by the National Institute for Health Research (NIHR) Biomedical Research Centre based at Guy's and St Thomas' NHS Foundation Trust and King's College London. In addition, M. Loda's work ML's work was supported by the NIH grants RO1CA131945, P01 CA08902, P50 CA90381, and the Prostate Cancer Foundation. The views expressed are those of the author(s) and not necessarily those of the NHS, the NIHR, the Department of Health, the NIH or the Prostate Cancer Foundation.

\section{References}

[1] S. M. Grundy, "Metabolic complications of obesity," Endocrine, vol. 13, no. 2, pp. 155-165, 2000.

[2] S. H. Jee, H. J. Kim, and J. Lee, "Obesity, insulin resistance and cancer risk,” Yonsei Medical Journal, vol. 46, no. 4, pp. 449-455, 2005.

[3] R. T. Jung, "Obesity as a disease," British Medical Bulletin, vol. 53, no. 2, pp. 307-321, 1997.

[4] J. R. Pender and W. J. Pories, "Epidemiology of obesity in the United States," Gastroenterology Clinics of North America, vol. 34, no. 1, pp. 1-7, 2005.

[5] V. J. Lawrence and P. G. Kopelman, "Medical consequences of obesity," Clinics in Dermatology, vol. 22, no. 4, pp. 296-302, 2004.

[6] H. Vainio, R. Kaaks, and F. Bianchini, "Weight control and physical activity in cancer prevention: international evaluation of the evidence," European Journal of Cancer Prevention, vol. 11, no. 2, pp. S94-S100, 2002.

[7] A. G. Renehan, M. Tyson, M. Egger, R. F. Heller, and M. Zwahlen, "Body-mass index and incidence of cancer: a systematic review and meta-analysis of prospective observational studies," The Lancet, vol. 371, no. 9612, pp. 569-578, 2008.

[8] S. D. H. Malnick and H. Knobler, "The medical complications of obesity," QJM, vol. 99, no. 9, pp. 565-579, 2006.
[9] A. A. Arslan, K. J. Helzlsouer, C. Kooperberg et al., "Anthropometric measures, body mass index, and pancreatic cancer: a pooled analysis from the pancreatic cancer cohort consortium (PanScan)," Archives of Internal Medicine, vol. 170, no. 9, pp. 791-802, 2010.

[10] T. B. Van Itallie, "Obesity: adverse effects on health and longevity," American Journal of Clinical Nutrition, vol. 32, no. 12, pp. 2723-2733, 1979.

[11] A. Vrieling, K. Buck, R. Kaaks, and J. Chang-Claude, "Adult weight gain in relation to breast cancer risk by estrogen and progesterone receptor status: a meta-analysis," Breast Cancer Research and Treatment, vol. 123, no. 3, pp. 641-649, 2010.

[12] D. Saunders, D. Seidel, M. Allison, and G. Lyratzopoulos, "Systematic review: the association between obesity and hepatocellular carcinoma-epidemiological evidence," Alimentary Pharmacology and Therapeutics, vol. 31, no. 10, pp. 1051-1063, 2010.

[13] B. F. Fuemmeler, M. K. Pendzich, and K. P. Tercyak, "Weight, dietary behavior, and physical activity in childhood and adolescence: implications for adult cancer risk," Obesity Facts, vol. 2, no. 3, pp. 179-186, 2009.

[14] D. J. Harriss, G. Atkinson, K. George et al., "Lifestyle factors and colorectal cancer risk (1): systematic review and meta-analysis of associations with body mass index," Colorectal Disease, vol. 11, no. 6, pp. 547-563, 2009.

[15] Z. Dai, Y. C. Xu, and L. Niu, "Obesity and colorectal cancer risk: a meta-analysis of cohort studies," World Journal of Gastroenterology, vol. 13, no. 31, pp. 4199-4206, 2007.

[16] S. C. Larsson and A. Wolk, "Obesity and colon and rectal cancer risk: a meta-analysis of prospective studies," American Journal of Clinical Nutrition, vol. 86, no. 3, pp. 556-565, 2007.

[17] Y. Qian and J. G. Fan, "Obesity, fatty liver and liver cancer," Hepatobiliary \& Pancreatic Diseases International, vol. 4, no. 2, pp. 173-177, 2005.

[18] D. Leroith, R. Novosyadlyy, E. J. Gallagher et al., "Obesity and type 2 diabetes are associated with an increased risk of developing cancer and a worse prognosis; epidemiological and mechanistic evidence," Experimental and Clinical Endocrinology and Diabetes, vol. 116, no. 1, pp. S4-S6, 2008.

[19] M. J. Khandekar, P. Cohen, and B. M. Spiegelman, "Molecular mechanisms of cancer development in obesity," Nature Reviews Cancer, vol. 11, no. 12, pp. 886-895, 2011.

[20] P. J. Meikle and M. J. Christopher, "Lipidomics is providing new insight into the metabolic syndrome and its sequelae," Current Opinion in Lipidology, vol. 22, no. 3, pp. 210-215, 2011.

[21] M. Tanasescu, M. F. Leitzmann, E. B. Rimm, and F. B. Hu, "Physical activity in relation to cardiovascular disease and total mortality among men with type 2 diabetes," Circulation, vol. 107, no. 19, pp. 2435-2439, 2003.

[22] T. M. Manini, J. E. Everhart, K. V. Patel et al., "Daily activity energy expenditure and mortality among older adults," Journal of the American Medical Association, vol. 296, no. 2, pp. 171-179, 2006.

[23] E. W. Gregg, J. A. Cauley, K. Stone et al., "Relationship of changes in physical activity and mortality among older women," Journal of the American Medical Association, vol. 289, no. 18, pp. 2379-2386, 2003.

[24] P. J. Elmer, E. Obarzanek, W. M. Vollmer et al., "Effects of comprehensive lifestyle modification on diet, weight, physical fitness, and blood pressure control: 18-Month results of a randomized trial," Annals of Internal Medicine, vol. 144, no. 7, pp. 485-495, 2006. 
[25] J. A. Meyerhardt, E. L. Giovannucci, S. Ogino et al., "Physical activity and male colorectal cancer survival," Archives of Internal Medicine, vol. 169, no. 22, pp. 2102-2108, 2009.

[26] A. McTiernan, M. Irwin, and V. VonGruenigen, "Weight, physical activity, diet, and prognosis in breast and gynecologic cancers," Journal of Clinical Oncology, vol. 28, no. 26, pp. 4074-4080, 2010.

[27] J. A. Ligibel, A. Giobbie-Hurder, D. Olenczuk et al., "Impact of a mixed strength and endurance exercise intervention on levels of adiponectin, high molecular weight adiponectin and leptin in breast cancer survivors," Cancer Causes \& Control, vol. 20, no. 8, pp. 1523-1528, 2009.

[28] J. A. Ligibel, N. Campbell, A. Partridge et al., "Impact of a mixed strength and endurance exercise intervention on insulin levels in breast cancer survivors," Journal of Clinical Oncology, vol. 26, no. 6, pp. 907-912, 2008.

[29] M. J. Gonyeau and D. W. Yuen, "A clinical review of statins and cancer: helpful or harmful?” Pharmacotherapy, vol. 30, no. 2, pp. 177-194, 2010.

[30] J. R. Emberson, P. M. Kearney, L. Blackwell, C. Newman, C. Reith, N. Bhala et al., "Lack of effect of lowering LDL cholesterol on cancer: meta-analysis of individual data from 175,000 people in 27 randomised trials of statin therapy," PloS One, vol. 7, no. 1, Article ID e29849, 2012.

[31] A. D. Mooradian, M. J. Haas, K. R. Wehmeier, and N. C. W. Wong, "Obesity-related changes in high-density lipoprotein metabolism," Obesity, vol. 16, no. 6, pp. 1152-1160, 2008.

[32] H. Jafri, A. A. Alsheikh-Ali, and R. H. Karas, "Baseline and Ontreatment high-density lipoprotein cholesterol and the risk of cancer in randomized controlled trials of lipid-altering therapy," Journal of the American College of Cardiology, vol. 55, no. 25, pp. 2846-2854, 2010.

[33] D. E. Kok, J. G. van Roermund, K. K. Aben, M. den Heijer, D. W. Swinkels, E. Kampman et al., "Blood lipid levels and prostate cancer risk, a cohort study," Prostate Cancer and Prostatic Diseases, vol. 14, no. 4, pp. 340-345, 2011.

[34] D. McAuley, “Conventional Units-International Units," 1993 , http://www.globalrph.com/conv_si.htm.

[35] I. Jungner, G. Walldius, I. Holme, W. Kolar, and E. Steiner, "Apolipoprotein B and A-I in relation to serum cholesterol and triglycerides in 43000 Swedish males and females," International Journal of Clinical \& Laboratory Research, vol. 21, no. 2-4, pp. 247-255, 1992.

[36] M. G. Shlipak, R. Katz, B. Kestenbaum et al., "Rate of kidney function decline in older adults: a comparison using creatinine and cystatin C," American Journal of Nephrology, vol. 30, no. 3, pp. 171-178, 2009.

[37] J. Millan, X. Pinto, A. Munoz, M. Zuniga, J. Rubies-Prat, L. F. Pallardo et al., "Lipoprotein ratios: physiological significance and clinical usefulness in cardiovascular prevention," Vascular Health and Risk Management, vol. 5, pp. 757-765, 2009.

[38] J. I. Cleeman, "Executive summary of the third report of the National Cholesterol Education Program (NCEP) expert panel on detection, evaluation, and treatment of high blood cholesterol in adults (adult treatment panel III)," Journal of the American Medical Association, vol. 285, no. 19, pp. 2486-2497, 2001.

[39] J. Ahn, U. Lim, S. J. Weinstein et al., "Prediagnostic total and high-density lipoprotein cholesterol and risk of cancer," Cancer Epidemiology Biomarkers and Prevention, vol. 18, no. 11, pp. 2814-2821, 2009.
[40] J. J. Deeks, D. G. Altman, and M. J. Bradburn, "Statistical Methods for Examining Heterogeneity and Combining Results from Several Studies in Meta-Analysis," in Systematic Reviews in Health Care: Meta-Analysis in Context, M. Egger, G. D. Smith, and D. G. Altman, Eds., Chapter 15, BMJ Publishing, London, UK, 2008.

[41] J. V. Swinnen, H. Heemers, T. Van De Sande et al., "Androgens, lipogenesis and prostate cancer," Journal of Steroid Biochemistry and Molecular Biology, vol. 92, no. 4, pp. 273-279, 2004.

[42] N. Mehta, J. Hordines, C. Volpe, R. Doerr, and S. A. Cohen, "Cellular effects of hypercholesterolemia in modulation of cancer growth and metastasis: a review of the evidence," Surgical Oncology, vol. 6, no. 3, pp. 179-185, 1997.

[43] R. M. Arliss and C. A. Biermann, "Do soy isoflavones lower cholesterol, inhibit atherosclerosis, and play a role in cancer prevention?" Holistic Nursing Practice, vol. 16, no. 5, pp. 40-48, 2002.

[44] M. Mutoh, T. Akasu, M. Takahashi et al., "Possible involvement of hyperlipidemia in increasing risk of colorectal tumor development in human familial adenomatous polyposis," Japanese Journal of Clinical Oncology, vol. 36, no. 3, pp. 166-171, 2006.

[45] R. Flavin, G. Zadra, and M. Loda, "Metabolic alterations and targeted therapies in prostate cancer," Journal of Pathology, vol. 223, no. 2, pp. 283-294, 2011.

[46] O. Micheau, E. Solary, A. Hammann, F. Martin, and M. T. Dimanche-Boitrel, "Sensitization of cancer cells treated with cytotoxic drugs to fas- mediated cytotoxicity," Journal of the National Cancer Institute, vol. 89, no. 11, pp. 783-789, 1997.

[47] T. Migita, S. Ruiz, A. Fornari et al., "Fatty acid synthase: a metabolic enzyme and candidate oncogene in prostate cancer," Journal of the National Cancer Institute, vol. 101, no. 7, pp. 519-532, 2009.

[48] S. Rossi, E. Graner, P. Febbo et al., "Fatty acid synthase expression defines distinct molecular signatures in prostate cancer," Molecular Cancer Research, vol. 1, no. 10, pp. 707-715, 2003.

[49] Y. Kimura and M. Sumiyoshi, "High-fat, high-sucrose, and high-cholesterol diets accelerate tumor growth and metastasis in tumor-bearing mice," Nutrition and Cancer, vol. 59, no. 2, pp. 207-216, 2007.

[50] M. Brown, C. Hart, T. Tawadros, V. Ramani, V. Sangar, M. Lau et al., "The differential effects of statins on the metastatic behaviour of prostate cancer," British Journal of Cancer, vol. 106, no. 10, pp. 1689-1696, 2012.

[51] Y. Yasuda, M. Shimizu, Y. Shirakami et al., "Pitavastatin inhibits azoxymethane-induced colonic preneoplastic lesions in C57BL/KsJ-db/db obese mice," Cancer Science, vol. 101, no. 7, pp. 1701-1707, 2010.

[52] E. J. Jacobs and S. M. Gapstur, "Cholesterol and cancer: answers and new questions," Cancer Epidemiology Biomarkers and Prevention, vol. 18, no. 11, pp. 2805-2806, 2009.

[53] L. Calabresi, G. Rossoni, M. Gomaraschi, F. Sisto, F. Berti, and G. Franceschini, "High-density lipoproteins protect isolated rat hearts from ischemia-reperfusion injury by reducing cardiac tumor necrosis factor- $\alpha$ content and enhancing prostaglandin release," Circulation Research, vol. 92, no. 3, pp. 330-337, 2003.

[54] C. Pichard, G. Plu-Bureau, M. Neves-e Castro, and A. Gompel, "Insulin resistance, obesity and breast cancer risk," Maturitas, vol. 60, no. 1, pp. 19-30, 2008.

[55] B. Adderley-Kelly and E. Williams-Stephens, "The relationship between obesity and breast cancer," The ABNF Journal, vol. 14, no. 3, pp. 61-65, 2003. 
[56] J. B. Barnett, "The relationship between obesity and breast cancer risk and mortality," Nutrition Reviews, vol. 61, no. 2, pp. 73-76, 2003.

[57] R. Kaaks, A. Lukanova, and M. S. Kurzer, "Obesity, endogenous hormones, and endometrial cancer risk: a synthetic review," Cancer Epidemiology Biomarkers and Prevention, vol. 11, no. 12, pp. 1531-1543, 2002.

[58] P. M. Bracci, "Obesity and pancreatic cancer: overview of epidemiologic evidence and biologic mechanisms," Molecular Carcinogenesis, vol. 51, no. 1, pp. 53-63, 2012.

[59] L. Jiao, A. Berrington de Gonzalez, P. Hartge, R. M. Pfeiffer, Y. Park, D. M. Freedman et al., "Body mass index, effect modifiers, and risk of pancreatic cancer: a pooled study of seven prospective cohorts," Cancer Causes \& Control, vol. 21, no. 8, pp. 1305-1314, 2010.

[60] A. B. De Gonzalez, S. Sweetland, and E. Spencer, "A metaanalysis of obesity and the risk of pancreatic cancer," British Journal of Cancer, vol. 89, no. 3, pp. 519-523, 2003.

[61] M. A. Moyad, "Is obesity a risk factor for prostate cancer, and does it even matter? A hypothesis and different perspective," Urology, vol. 59, no. 4, pp. 41-50, 2002.

[62] "Definition and diagnosis of diabetes mellitus and intermediate hyperglycemia," Report of A WHO/IDF Consulation, World Health Organisation, Geneva, Switzerland, 2006.

[63] M. Inoue, M. Noda, N. Kurahashi et al., "Impact of metabolic factors on subsequent cancer risk: results from a large-scale population-based cohort study in Japan," European Journal of Cancer Prevention, vol. 18, no. 3, pp. 240-247, 2009.

[64] H. Iso, A. Ikeda, M. Inoue, S. Sato, and S. Tsugane, "Serum cholesterol levels in relation to the incidence of cancer: the JPHC study cohorts," International Journal of Cancer, vol. 125, no. 11, pp. 2679-2686, 2009.

[65] C. M. Kitahara, A. B. De González, N. D. Freedman et al., "Total cholesterol and cancer risk in a large prospective study in Korea," Journal of Clinical Oncology, vol. 29, no. 12, pp. 1592-1598, 2011.

[66] A. G. Mainous III, B. J. Wells, R. J. Koopman, C. J. Everett, and J. M. Gill, "Iron, lipids, and risk of cancer in the Framingham offspring cohort," American Journal of Epidemiology, vol. 161, no. 12, pp. 1115-1122, 2005.

[67] J. C. Melvin, D. Seth, L. Holmberg et al., "Lipid profiles and risk of breast and ovarian cancer in the Swedish AMORIS study," Cancer Epidemiology, Biomarkers \& Prevention, vol. 21, no. 8, pp. 1381-1384, 2012.

[68] S. J. Chang, M. F. Hou, S. M. Tsai et al., "The association between lipid profiles and breast cancer among Taiwanese women," Clinical Chemistry and Laboratory Medicine, vol. 45, no. 9, pp. 1219-1223, 2007.

[69] A. S. Furberg, M. B. Veierød, T. Wilsgaard, L. Berstein, and I. Thune, "Serum high density lipoprotein cholesterol, metabolic profile, and breast cancer risk," Journal of the National Cancer Institute, vol. 96, no. 15, pp. 1152-1160, 2004.

[70] M. Ha, J. Sung, and Y. M. Song, "Serum total cholesterol and the risk of breast cancer in postmenopausal Korean women," Cancer Causes \& Control, vol. 20, no. 7, pp. 1055-1060, 2009.

[71] N. Hayashi, M. Matsushima, T. Yamamoto, H. Sasaki, H. Takahashi, and S. Egawa, "The impact of hypertriglyceridemia on prostate cancer development in patients aged $\geq 60$ years," $B J U$ International, vol. 109, no. 4, pp. 515-519, 2012.

[72] J. A. Kaye, C. R. Meier, A. M. Walker, and H. Jick, "Statin use, hyperlipidaemia, and the risk of breast cancer," British Journal of Cancer, vol. 86, no. 9, pp. 1436-1439, 2002.
[73] Y. Kim, S. K. Park, W. Han et al., "Serum high-density lipoprotein cholesterol and breast cancer risk by menopausal status, body mass index, and hormonal receptor in Korea," Cancer Epidemiology Biomarkers \& Prevention, vol. 18, no. 2, pp. 508-515, 2009.

[74] K. Lindemann, L. J. Vatten, M. Ellstrøm-Engh, and A. Eskild, "Serum lipids and endometrial cancer risk: results from the HUNT-II study," International Journal of Cancer, vol. 124, no. 12, pp. 2938-2941, 2009.

[75] A. E. Cust, R. Kaaks, C. Friedenreich et al., "Metabolic syndrome, plasma lipid, lipoprotein and glucose levels, and endometrial cancer risk in the European Prospective Investigation into Cancer and Nutrition (EPIC)," Endocrine-Related Cancer, vol. 14, no. 3, pp. 755-767, 2007.

[76] D. Seth, H. Garmo, A. Wigertz et al., "Lipid profiles and the risk of endometrial cancer in the Swedish AMORIS study," International Journal of Molecular Epidemiology and Genetics, vol. 3, no. 2, pp. 122-133, 2012.

[77] L. Magura, R. Blanchard, B. Hope, J. R. Beal, G. G. Schwartz, and A. E. Sahmoun, "Hypercholesterolemia and prostate cancer: a hospital-based case-control study," Cancer Causes \& Control, vol. 19, no. 10, pp. 1259-1266, 2008.

[78] A. M. Mondul, S. L. Clipp, K. J. Helzlsouer, and E. A. Platz, "Association between plasma total cholesterol concentration and incident prostate cancer in the CLUE II cohort," Cancer Causes and Control, vol. 21, no. 1, pp. 61-68, 2010.

[79] A. M. Mondul, S. J. Weinstein, J. Virtamo, and D. Albanes, "Serum total and HDL cholesterol and risk of prostate cancer," Cancer Causes \& Control, vol. 22, no. 11, pp. 1545-1552, 2011.

[80] E. A. Platz, C. Till, P. J. Goodman et al., "Men with low serum cholesterol have a lower risk of high-grade prostate cancer in the placebo arm of the prostate cancer prevention trial," Cancer Epidemiology Biomarkers \& Prevention, vol. 18, no. 11, pp. 2807-2813, 2009.

[81] M. Van Hemelrijck, G. Walldius, I. Jungner et al., "Low levels of apolipoprotein A-I and HDL are associated with risk of prostate cancer in the Swedish AMORIS study," Cancer Causes \& Control, vol. 22, no. 7, pp. 1011-1019, 2011.

[82] M. Van Hemelrijck, H. Garmo, L. Holmberg et al., "Prostate cancer risk in the Swedish AMORIS study," Cancer, vol. 117, no. 10, pp. 2086-2095, 2011.

[83] F. Bravi, L. Scotti, C. Bosetti et al., "Self-reported history of hypercholesterolaemia and gallstones and the risk of prostate cancer," Annals of Oncology, vol. 17, no. 6, pp. 1014-1017, 2006.

[84] M. Tsushima, A. M. Y. Nomura, J. Lee, and G. N. Stemmermann, "Prospective study of the association of serum triglyceride and glucose with colorectal cancer," Digestive Diseases and Sciences, vol. 50, no. 3, pp. 499-505, 2005.

[85] F. J. van Duijnhoven, H. B. Bueno-De-Mesquita, M. Calligaro et al., "Blood lipid and lipoprotein concentrations and colorectal cancer risk in the European Prospective Investigation into Cancer and Nutrition," Gut, vol. 60, no. 8, pp. 1094-1102, 2011.

[86] Y. W. Chung, D. S. Han, Y. K. Park et al., "Association of obesity, serum glucose and lipids with the risk of advanced colorectal adenoma and cancer: a case-control study in Korea," Digestive and Liver Disease, vol. 38, no. 9, pp. 668-672, 2006.

[87] M. Van Hemelrijck, H. Garmo, N. Hammar et al., “The interplay between lipid profiles, glucose, BMI and risk of kidney cancer in the Swedish AMORIS study," International Journal of Cancer, vol. 130, no. 9, pp. 2118-2128, 2012. 
[88] G. Andreotti, J. Chen, Y.-T. Gao et al., "Serum lipid levels and the risk of biliary tract cancers and biliary stones: A populationbased study in China," International Journal of Cancer, vol. 122, no. 10, pp. 2322-2329, 2008.

[89] K. Asano, M. Kubo, K. Yonemoto et al., "Impact of serum total cholesterol on the incidence of gastric cancer in a populationbased prospective study: the Hisayama study," International Journal of Cancer, vol. 122, no. 4, pp. 909-914, 2008. 


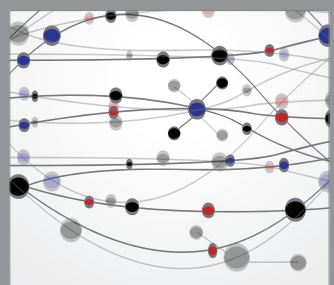

The Scientific World Journal
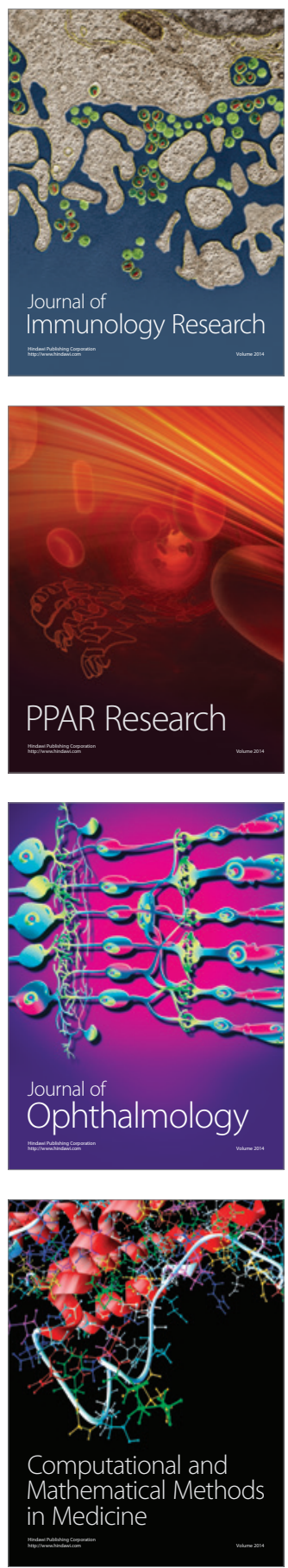

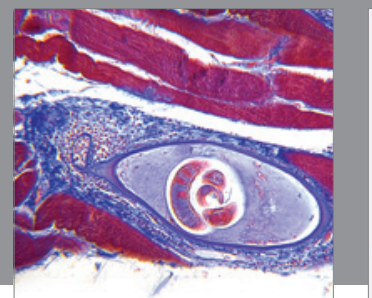

Gastroenterology

Research and Practice
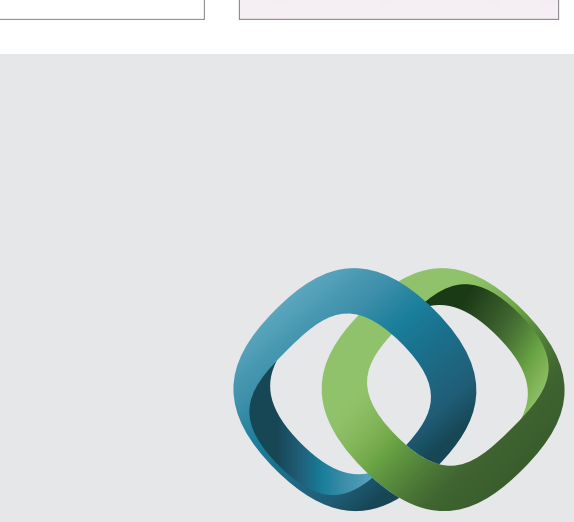

\section{Hindawi}

Submit your manuscripts at

http://www.hindawi.com
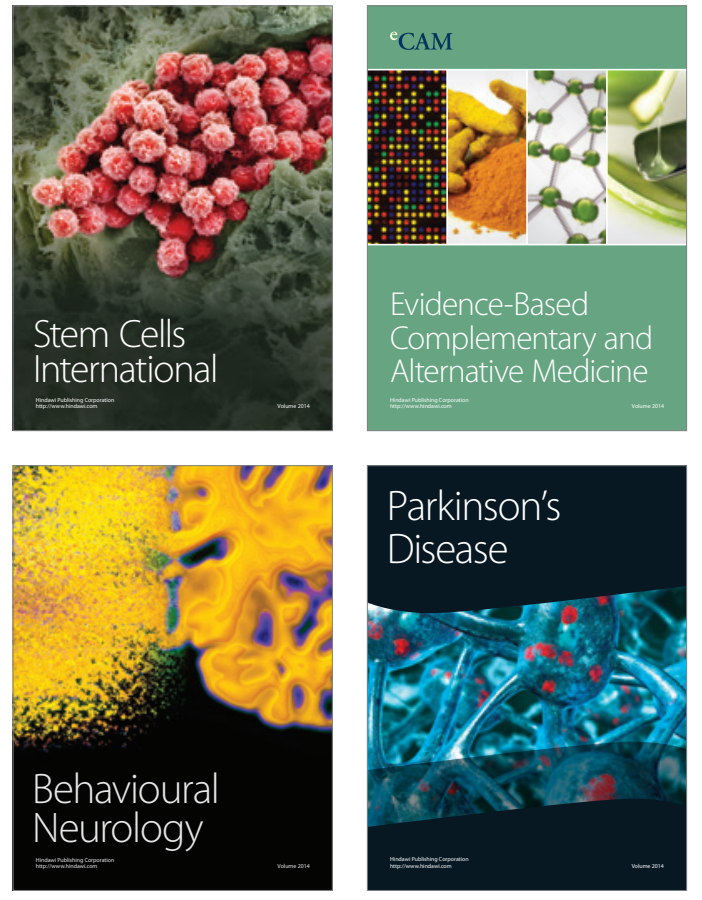
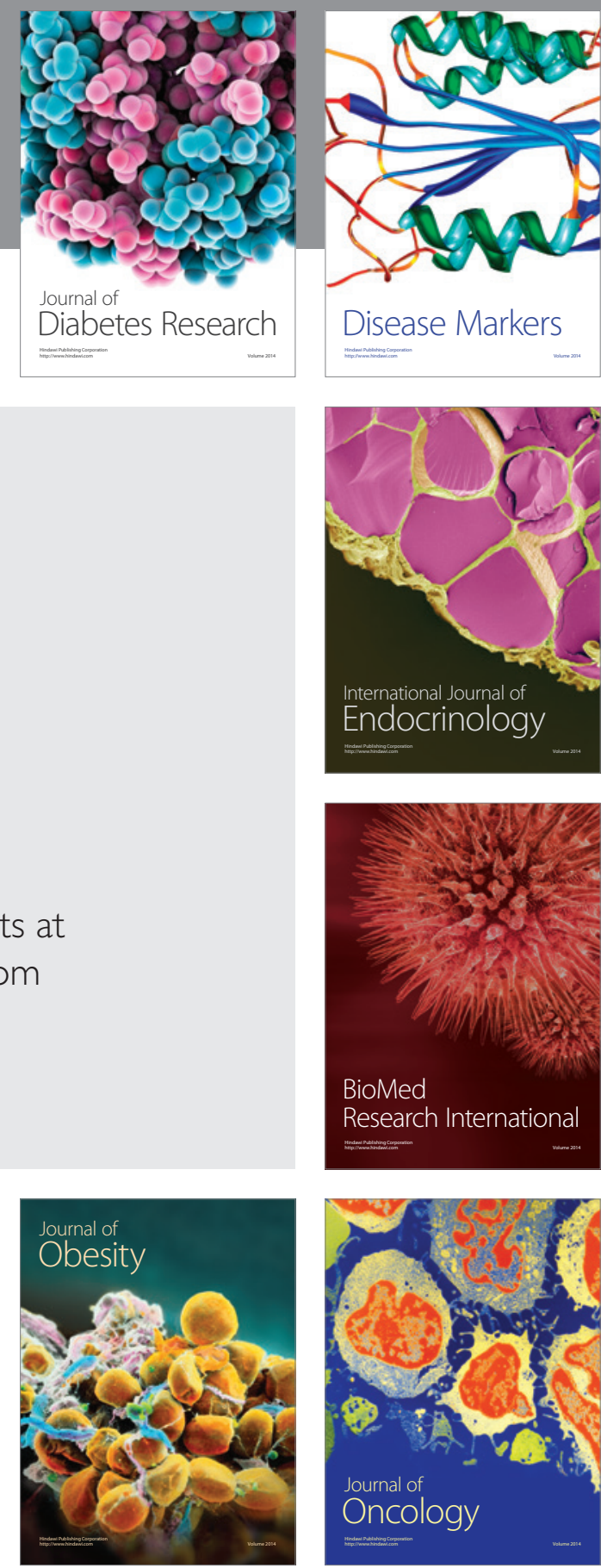

Disease Markers
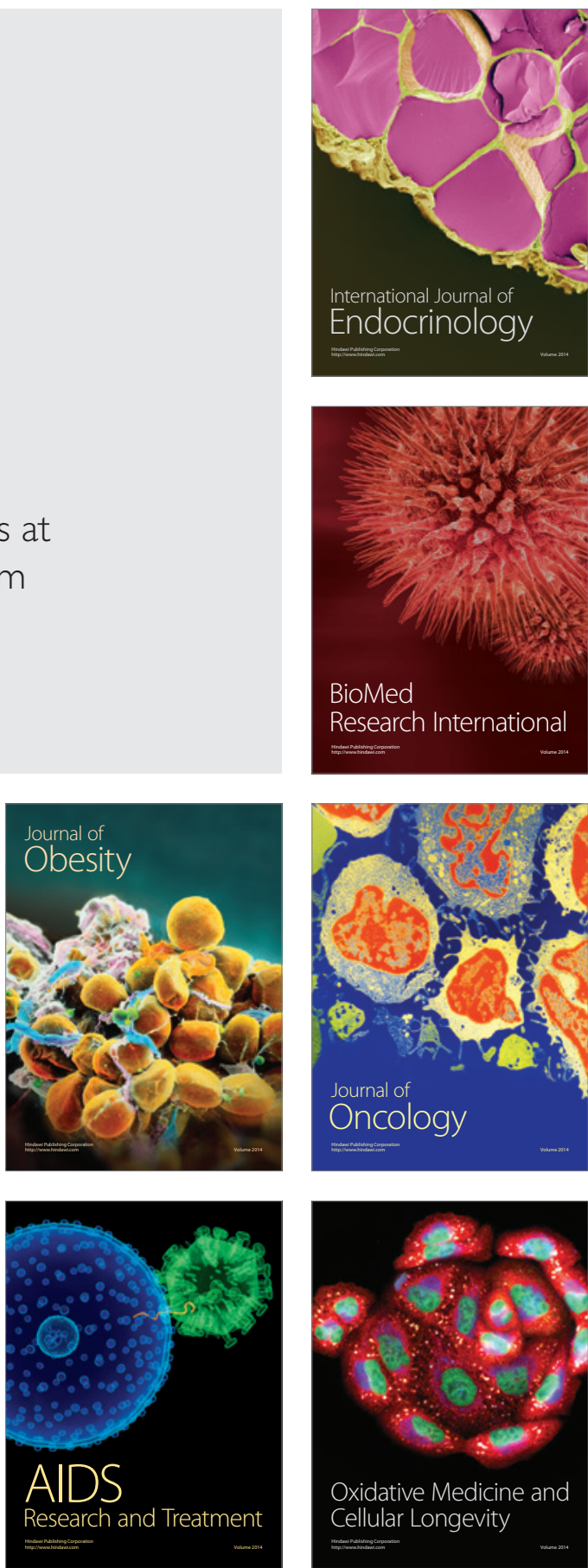